\title{
Severe bronchopulmonary dysplasia: outcomes before and after the implementation of an inpatient multidisciplinary team
}

\author{
Robin L. McKinney $\mathbb{D}^{1} \cdot$ Joseph J. Schmidhoefer ${ }^{1} \cdot$ Alyssa L. Balasco $^{1} \cdot$ Jason T. Machan $^{2} \cdot$ Priya Hirway $^{1} \cdot$ \\ Martin Keszler $\mathbb{D}^{1}$
}

Received: 8 April 2020 / Revised: 1 September 2020 / Accepted: 8 October 2020 / Published online: 23 October 2020

(c) The Author(s), under exclusive licence to Springer Nature America, Inc. 2020

\begin{abstract}
Objective Severe bronchopulmonary dysplasia (sBPD) can lead to long term morbidity. We created a sBPD multidisciplinary team in 2011 to optimize care and improve outcomes.

Study design Retrospective chart review of three groups between 2008 and 2016: patients with sBPD born before 2011, patients with sBPD born after 2011, and patients with moderate BPD born after 2011.

Results Infants with SBPD after 2011 had a shorter NICU length of stay compared with children born before 2011 (mean 140 days vs 170 days $p<0.007$ ), weighed more at discharge ( $\mathrm{z}$-score $-0.8 \mathrm{vs}-1.35 p=0.01$ ), had less failure to thrive post discharge $(32 \%$ vs $51 \% p=0.05)$ and had more well visits in the first six months of life (mean 6.7 vs $5.3 p=0.04$ ). No difference was observed in the rate of readmissions in the first two years of life.

Conclusion Our multidisciplinary team has improved the inpatient management of patients with sBPD.
\end{abstract}

\section{Introduction}

Bronchopulmonary dysplasia (BPD) due to prematurity is one of the most common chronic lung diseases affecting children today and the most common late morbidity of preterm birth $[1,2]$. With advances in obstetrical and neonatal management, the advent of surfactant, more sophisticated ventilators and increased survival of children born at the extremes of viability and birth weight, the prevalence of severe bronchopulmonary dysplasia (sBPD) has been increasing [1-4]. Nationally the incidence of SBPD has been reported as $16 \%$ for all children born $<32$ weeks [5-9]. Using this estimate there are approximately 13,000 new patients across the United States who develop sBPD every year [5-9]. Children with SBPD are at greater risk for many co-morbid conditions: gastro-esophageal reflux, failure to

Robin L. McKinney

Robin_mckinney@brown.edu

1 Department of Pediatrics, The Warren Alpert School of Medicine at Brown University, Providence, RI, USA

2 Lifespan Biostatistics Core and Departments of Orthopedics and Surgery, Warren Alpert Medical School, Brown University, Providence, RI, USA thrive (FTT), pulmonary hypertension, asthma, tracheobroncho malacia, tracheostomy, ventilator dependence and neurodevelopmental delays, to name a few. The majority of neonatal research has focused on strategies aimed at preventing BPD, but there is a paucity of research on the care and best practice management in children who have already developed BPD.

The 2001 National Institute of Child Health and Human Development (NICHD) definition of BPD uses the level of respiratory support patients are receiving at 36 weeks to divide BPD into three separate categories (mild, moderate, severe) [10]. Because this definition uses respiratory support, the focus of BPD research has been on the lungs. However, children with BPD have multiple organ system involvement, and children with SBPD carry the greatest burden of morbidity and mortality. The multiple comorbidities result in the involvement of multiple subspecialists, potentially leading to miscommunication between care teams and poor continuity of care. It has been proposed that an interdisciplinary team made up of multiple subspecialists dedicated to the care of infants with SBPD should be utilized to improve outcomes [11]. In November of 2011 we created a multidisciplinary severe Bronchopulmonary Dysplasia Team (BIT:s) consisting of a pediatric pulmonologist, cardiologist, intensivist, pharmacist and a neonatologist with an interest in BPD. A dedicated pediatric 
ear, nose and throat surgeon and later gastroenterologist were available to consult based on criteria developed by the team. Referral to BIT:s was based on the diagnosis of severe BPD per the 2001 NICHD definition of BPD and broadened to include patients we agreed would benefit from the care of this multidisciplinary team. To test the effectiveness of our team we performed a retrospective review of patients referred to BIT:s compared with historical controls of patients with SBPD who would have met the criteria for referral to BIT:s had the team been in existence. We included infants with BPD who did not meet the criteria for inclusion in the BIT:s service as a second reference cohort of BPD infants with less severe disease.

\section{Methods}

Electronic medical records were reviewed from 2008-2016 to identify infants with a diagnosis of moderate to severe BPD using the NICHD definition. Infants were classified as either: (1) patients referred to our multidisciplinary BIT:s team (created November 2011), (2) a cohort of patients meeting the inclusion/exclusion criteria for BIT:s referral born before the program's inception, or (3) patients who met the NICHD criteria for moderate BPD born after 2011, and did not meet the criteria for referral to BIT:s. The BIT:s and Pre-BIT:s groups were matched for disease severity at 36 weeks PMA based off the NICHD definition of sBPD.

The criteria for referral to the BIT:s team are infants with a postmenstrual age $\geq 36$ weeks and any of the following: pulmonary hypertension, on non-invasive positive pressure ventilation, on continuous positive airway pressure, high flow nasal cannula $\geq 2 \mathrm{~L} / \mathrm{min}$, or $\mathrm{FiO}_{2}>0.3$. Patients were referred to the BIT:s team at 36 weeks PMA.. 36 weeks PMA was used as the comparison point for disease severity because regardless of birth characteristics BPD is defined at 36 weeks PMA. Patients were excluded if they were referred to the BIT:s team, but did not meet the criteria for SBPD, were lost to follow up before their first outpatient appointment, or if they had less than 1.5 years of follow data available from the date of their birth within the study period.

All infants were born at Women and Infants Hospital, or transferred to Women and Infants Hospital within the first $12 \mathrm{~h}$ of life. Women and Infants is a tertiary care academic NICU and the only level 4 NICU in the state. All premature and high risk pregnancies in the state are referred to Women and Infants. There are no infants less than 32 weeks who require respiratory support cared for anywhere else in the state. There are approximately 9000 deliveries, at our hospital and 140 admissions to the NICU of infants born less than 32 weeks per year. Between 2012 and 2017 an average of 24 patients per year were diagnosed with sBPD all of whom were referred to the BIT:s team. All follow up visits, subspecialty visits and readmissions occurred either at Women and Infants Hospital or Hasbro Children's Hospital. There are no other hospitals within the state that offer subspecialty care, and no other hospitals have a pediatric ED or pediatric intensive care unit. All young pediatric patients are referred to Hasbro Children's Hospital.

The BIT:s team met and rounded on all referred patients every two weeks with the primary NICU team. At the time referral (36wks PMA), a screening echocardiogram for pulmonary hypertension and structural anomalies was performed on all patients regardless of any previous echocardiograms. If no pulmonary hypertension was identified, then an echocardiogram was performed either just before discharge or any time the patient had significant clinical deterioration. If pulmonary hypertension was identified recommendations were made to have $\mathrm{O}_{2}$ saturations maintained above $96 \%$, to optimize respiratory support. Medications (inhaled nitric oxide, sildenafil or diuretics) were started as clinically indicated. One patient with significant pulmonary hypertension was referred for cardiac catheterization and found to have pulmonary vein stenosis. Right ventricular (RV) systolic pressure was assessed by peak tricuspid regurgitant (TR) doppler velocity plus estimated right atrial pressure, or peak Doppler velocity across a patent ductus arteriosus or ventricular septal defect if present. If there were inadequate TR envelopes, the interventricular end-systolic septal position was assessed in high parasternal short axis view. Pulmonary pressures based on the position of the septum were estimated as follows: fully rounded left ventricle (LV) suggested estimated RV systolc pressure less than or equal to half systemic pressure, if flattened estimated RV systolic pressure $50-100 \%$ of systemic pressure was assumed, dependent on degree of flattening. If the septum was concave (bowing into the LV) estimated RV systolic pressure was supra-systemic. In all cases the presence of RV hypertrophy, RV dilation, RV function, Doppler pattern of right heart outflow tract flow, main pulmonary artery size and direction and amount of shunting across the interatrial septum were also assessed as corroborating evidence if interventricular septal position was used as a primary assessment [12]. All echocardiograms were reviewed by the cardiologist on the BIT:s team who has a special interest in pulmonary hypertension and the findings relayed to the primary team.

Recommendations regarding respiratory support were standardized with an emphasis on optimally supporting the patient vs rapid weaning from respiratory support. For intubated patients a high PEEP (typically greater than $10 \mathrm{cmH}_{2} \mathrm{O}$ ), long inspiratory time (0.6 s or longer), high tidal volume $(8-12 \mathrm{~mL} / \mathrm{kg}$ ) and low rate $(20-30 \mathrm{bpm})$ were recommended with an emphasis on weaning the PEEP slowly typically no more than by $1 \mathrm{cmH}_{2} \mathrm{O}$ per week while 
monitoring the patients spontaneous respiratory rate, $\mathrm{O}_{2}$ saturations, weight gain and length. Using these parameters to guide recommendations, ventilator settings were adjusted to promote growth as measured by weekly weights and monthly lengths. Tracheostomy was recommended when patients required high ventilator settings beyond 40-42 wks PMA and prolonged need for distending pressure was anticipated. Patients on non-invasive positive pressure ventilation (nasal CPAP, or high flow nasal cannula) were also weaned slowly with an emphasis on adequate support vs rapid weaning. The use of steroids and diuretics were discussed in the context of each individual patient and recommended as clinically indicated in cases where weaning had stalled. The need for optimal nutritional support while avoiding excessive calories and carbohydrate were always emphasized.

Patients with a weak cry, hoarse voice or inability to wean from support were evaluated by ENT for airway malacia or other problems. Patients with poor weight gain (Weight z-score below-1.5@36weeks PMA, or a decrease in $\mathrm{z}$-score greater than 0.8 from birth), feeding dysphagia with a gastrostomy tube or on anti-reflux medications were referred to gastroenterology and their feeding team prior to discharge. Gastroesophageal reflux was diagnosed using an impedance study with $\mathrm{pH}$ probe. Significant reflux was defined as 100 episodes $/ 24 \mathrm{~h}$ or more [13].

Demographic data including the patient's gestational age at birth, age at re-admission and outpatient visits, sex, growth chart, past medical history, surgical history, and specific medical history that pertains to bronchopulmonary dysplasia were extracted from the electronic medical records at both Women and Infants Hospital and Hasbro Children's Hospital. Number of hospital admissions, reasons for admission, emergency department (ED) visits, and co-morbidities such as pulmonary hypertension, asthma, albuterol use, failure to thrive (FTT) or feeding difficulties, Gastro-esophageal reflux, gastrostomy tube, nasogastric tube placement, tracheostomy placement were also recorded. Data on readmissions, well and ED visits were collected from discharge until the child was five years of age, or until January 1st, 2016, whichever came first.

\section{Statistical methods}

SAS version 9.4 (The SAS Institute, Cary, NC) was used for all analyses. Generalized linear models (proc glimmix) were constructed to compare patients falling into the three classifications of patient types. Patients who met the criteria for BIT:s referral prior to program inception represented a control for the BIT:s patients in reference to improvement, while patients with less severe BPD were included as a reference for other infants receiving treatment in the NICU concurrent with BIT:s. The distribution for each generalized model was chosen based on the nature of the dependent variable and examination of model residuals where appropriate. Classical sandwich estimation was applied to adjust for model misspecification (e.g., differences in variances not explained by the distribution chosen). Raw $p$ values comparing the cohorts were reported and used to draw conclusions along with raw $95 \%$ confidence intervals.

This study was approved and overseen by the Institutional review board of both institutions with waiver of consent.

\section{Results}

Demographic data for the 143 infants included in this analysis are shown in Table 1. There was no difference in birth characteristics between the three groups. There was no difference between the BIT:s and Pre-BIT:s groups in terms of the respiratory support they were receiving at 36 weeks PMA. Infants enrolled in the BIT:s program had significantly shorter average lengths of stay than the infants with SBPD born prior to the creation of BIT:s (140 days vs. 170 days, $p<0.007)$ and were discharged from the hospital at a significantly earlier postmenstrual age (46 weeks vs. 50 weeks, $p=0.01$ ). Infants enrolled in BIT:s had significantly better weight gain based on their z-score at discharge ( -0.8 vs $-1.35 p=0.02)$ than the pre-BIT:s group, but their $\mathrm{z}$-score for length $(-1.78 \mathrm{vs}-2.31 p=0.15)$ at time of discharge, though somewhat improved was not statistically different (Table 2).

Post discharge there was no difference in the time to first admission or the percentage of patients that were admitted after discharge from the NICU, between the Pre-BIT:s, BIT:s and $\mathrm{mBPD}$ groups in the first two years of life $(61 \%, 55$ and $53 \%$ respectively, Fig. 1a). At least one ED visit occurred in $71 \%$ of BIT:s patients, $65 \%$ of patients with $\mathrm{mBPD}$ and $53 \%$ of patients born pre-BIT:S (Fig. 1b). The BIT:s patients visited the ED sooner after discharge in their first two years of life than pre-BIT:s patients. This was statistically significant between the BIT:s and Pre-BIT:s group $(p=0.02)$, but not between mBPD patients and BIT:s or mBPD and pre-BIT:s patients ( $p=0.1$ and 0.4 Fig. 1b).

The average number of admissions and ED visits was not significantly different within the first 6 months after discharge between the groups $(p=0.27$ and $p=0.43$, respectively Table 3). Patients in the BIT:s group had significantly more well visits in the first six months after discharge than those in the pre-BIT:s group (mean of 6.7 vs $5.3, p=0.04$ Table 3 ).

Patients in the BIT:s group were less likely to be discharged home on oxygen than patients in the pre-BIT:s group $(70 \%$ vs $92 \% p=0.017)$. Patients in the BIT:s group 
Table 1 Demographic characteristics of patients in the three groups.

\begin{tabular}{lllll}
\hline & BIT:s $(n=69)$ & Pre-BIT:s $(n=37)$ & mBPD $(n=37)$ & $p$ value \\
\hline Birthweight $(\mathrm{gm})$ & $817(739-895)$ & $774(674-875)$ & $791(729-853)$ & 0.8154 \\
Gestational age (wks) & $25.8(25.4-26.2)$ & $25.8(25-26.6)$ & $25.8(25.3-26.3)$ & 0.998 \\
$\%$ Male $(n)$ & $64 \%$ & $59 \%$ & $46 \%$ & 0.179 \\
\hline
\end{tabular}

Values are means and $95 \%$ confidence intervals.

$P$ values represent a three way comparison between the groups using ANOVA.

\begin{tabular}{|c|c|c|c|c|}
\hline & $\begin{array}{l}\text { BIT:s } \\
N=69\end{array}$ & $\begin{array}{l}\text { Pre-BIT:s } \\
N=37\end{array}$ & $\begin{array}{l}\mathrm{mBPD} \\
N=37\end{array}$ & $\begin{array}{l}p \text { value BIT:s vs } \\
\text { Pre-BIT:s }\end{array}$ \\
\hline Mean NICU LOS (days) & 140 (CI 131-149) & $170(150-192)$ & $\begin{array}{l}109 \text { (CI } 102 \\
-116)\end{array}$ & 0.007 \\
\hline PMA at discharge (wks) & $46(44-47)$ & $50(47-53)$ & $41(40-42)$ & 0.01 \\
\hline Weight at discharge $\mathrm{z}$-score & $\begin{array}{l}-0.80(-1.03 \\
\text { to }-0.57)\end{array}$ & $\begin{array}{l}-1.35(-1.72 \\
\text { to }-0.98)\end{array}$ & $\begin{array}{l}-0.73(-1.07 \\
\text { to }-0.40)\end{array}$ & 0.02 \\
\hline Length at discharge $\mathrm{z}$-score & $\begin{array}{l}-1.78(-2.19 \\
\text { to }-1.37)\end{array}$ & $\begin{array}{l}-2.31(-2.90 \\
\text { to }-1.72)\end{array}$ & $\begin{array}{l}-1.32(-1.77 \\
\text { to }-0.88)\end{array}$ & 0.15 \\
\hline
\end{tabular}

$\mathrm{P}$ values represent comparison between BIT:s and Pre-BIT:s.
Table 2 Mean NICU length of stay (LOS), postmenstrual age (PMA) at discharge and corrected Z-score weight and length at time of discharge between the three groups with $95 \%$ confidence intervals. of stay of infants with sBPD by an average of 30 days without putting them at increased risk for readmission. Interestingly while these patients went home earlier, a lower percentage of them went home with oxygen than patients in the pre-BIT:s group. This could represent a change in practice over time, but if this were the case we would expect their length of stay to be longer while they were weaned off $\mathrm{O}_{2}$ in the hospital prior to discharge. There was also a 50\% reduction in tracheostomies at discharge from the NICU. This did not reach statistical significance but may be explained by the small sample size. The trend cannot be explained by a difference in the severity of BPD between the groups as they were matched for disease severity at 36 weeks PMA using the NICHD definition of sBPD. All interventions by the multi-disciplinary team were targeted after 36 weeks PMA with the goal of providing better support. Earlier discharge cannot be explained by the increased use of tracheostomies because the number of tracheostomies was less in the BIT:s group. This may represent a potential benefit of the multidisciplinary team recommending more optimal and timely respiratory support leading to shorter lengths of stay.

Most neonatologists and neonatology research focus on preventing BPD with less attention being paid to best practices once BPD has developed. Because severe BPD is relatively uncommon, the evidence base for care is incomplete. Thus, many aspects of the care of infants with SBPD may be poorly understood by practicing neonatologists. For example, respiratory support for this chronic condition still often follows the acute care model with different treatment paradigms [11]. Creating a multidisciplinary BPD team brings diverse expertise, familiarity with available literature 
and perhaps most importantly, personal commitment to the care of this unique and challenging patient population. Other strengths of the multidisciplinary approach are the
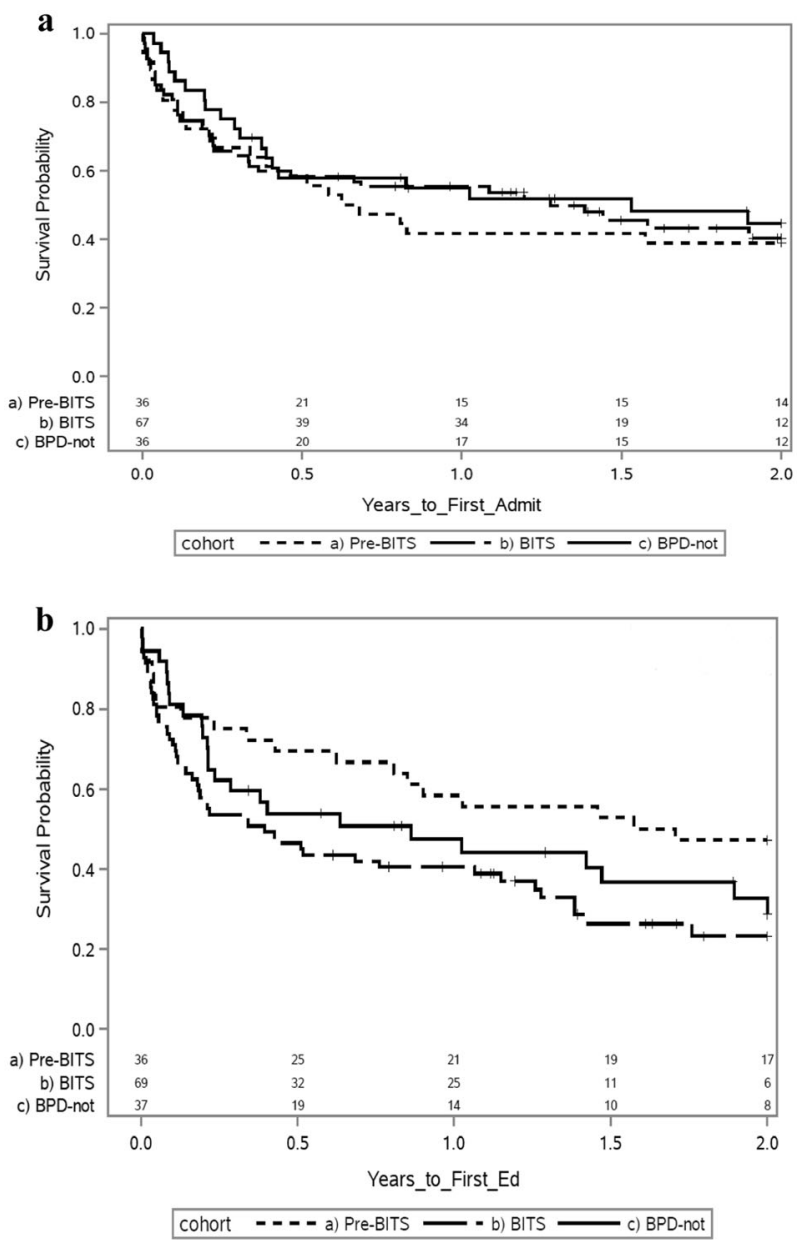

Fig. 1 Kaplan-Meier curves showing the time to first admission and ED visit. a The percentage and timing of 1 st admissions in the first two years of life between Pre-BIT:s, BIT:s and mBPD $(61 \%, 55$ and $53 \%$ respectively). There was no difference in the percentage or timing of admissions between BIT:s and Pre-BIT:s $(p=0.86)$. b The percentage and timing of 1 st ED visits in the first two years of life between Pre-BIT:s, BIT:s and mBPD (53\%, 71 and 65\%, respectively). There was a difference in the percentage and timing of ED visits between BIT:s and Pre-BIT:S $(p=0.02)$. standardization of practice at our institution across multiple providers and creating continuity of care with subspecialists from inpatient to outpatient. Our findings suggest that by standardizing the care across the different NICU providers and rounding with multiple specialists with clinical expertise in SBPD we have been able to improve the care of these infants with a substantial reduction in length of stay that should translate into reduced cost of hospitalization.

We also documented a rate of pulmonary hypertension that was half of what was seen in the pre-BIT:s era. This difference did not reach statistical significance, but may reflect the focus of the BIT:s team on optimizing respiratory care and avoiding factors known to increase the risk of pulmonary hypertension. Some of the strategies recommended by the BIT:s team were higher oxygen saturation targets (95-98\%) for patients with identified pulmonary hypertension and early adoption of chronic ventilation strategies that emphasized providing adequate support of the patient so that they could grow, both in length and weight, and avoiding rapid weaning aimed at removing support and discharging the patient home. More complete ascertainment of pulmonary hypertension in the BIT:s patients may have contributed to the lack of statistical significance, as echocardiograms were not universally done prior to the advent of BIT:s, whereas every patient enrolled with BIT:s received a screening echocardiogram for pulmonary hypertension.

We expected to find that the increased outpatient referral to specialists was associated with fewer emergency department (ED) visits, but this wasn't the case. BIT:s patients had more ED visits than the pre-BIT:s group. Interestingly, patients in the moderate BPD group had more ED visits than the pre-BIT:s group too. Ehrenkranz et al. showed that the rates of re-hospitalization are lower in moderate BPD compared to SBPD so it was surprising to see an increase use of the ED in both the moderate BPD and BIT:s groups [9]. Given that this study is comparing patients from two different time periods this may represent the national trend of increased emergency department utilization for low acuity problems, previously cared for in outpatient doctor's offices [15].
Table 3 Average number of admissions, ED visits and well visits in the first 6 months after discharge from the NICU with $95 \%$ confidence intervals.

\begin{tabular}{lllll}
\hline & $\begin{array}{l}\text { BIT:s } \\
N=69\end{array}$ & $\begin{array}{l}\text { Pre-BIT:s } \\
N=37\end{array}$ & $\begin{array}{l}\text { mBPD } \\
N=37\end{array}$ & $\begin{array}{l}p \text { value BIT:s } \\
\text { vs Pre-BIT:s }\end{array}$ \\
\hline $\begin{array}{l}\text { Mean number of admissions in } \\
\text { 1st } 6 \text { mo after discharge }\end{array}$ & $0.74(0.52-1.03)$ & $1.03(0.64-1.66)$ & $0.57(0.38-0.86)$ & 0.27 \\
$\begin{array}{l}\text { Average number of ED visits in } \\
\text { 1st 6 mo after discharge }\end{array}$ & $1.05(0.78-1.44)$ & $0.81(0.45-1.45)$ & $0.78(0.52-1.18)$ & 0.43 \\
$\begin{array}{l}\text { Mean well visits in first 6 mo } \\
\text { after discharge }\end{array}$ & $6.74(5.76-7.89)$ & $5.27(4.46-6.23)$ & $3.57(2.90-4.38)$ & 0.04 \\
\hline
\end{tabular}

There was no difference in the number of admissions or ED visits between the three groups, but BIT:S had significantly more well visits compared to Pre-BIT:S $(p=0.04)$. 
Table 4 Percentages and 95\% confidence intervals of comorbid conditions between the three groups.

\begin{tabular}{lllll}
\hline & BIT:s (\%) & Pre-BIT:s (\%) & mBPD (\%) & $\begin{array}{l}p \text { value } \\
\text { BIT:s vs Pre-BIT:s }\end{array}$ \\
\hline Discharged home on $\mathrm{O}_{2}$ & $70 \%(59-80)$ & $92 \%(83-100)$ & & 0.0175 \\
Tracheostomy & $13 \%(7-23)$ & $27 \%(15-44)$ & $3 \%(0.4-17)$ & 0.08 \\
PHTN & $15 \%(8-25)$ & $27 \%(15-44)$ & $5 \%(1-19)$ & 0.12 \\
GER & $62 \%(50-73)$ & $68 \%(21-81)$ & $38 \%(24-54)$ & 0.59 \\
Asthma & $20 \%(12-32)$ & $38 \%(24-54)$ & $22 \%(11-38)$ & 0.06 \\
Albuterol & $64 \%(52-74)$ & $65 \%(48-79)$ & $43 \%(28-60)$ & 0.91 \\
G-Tube & $25 \%(16-36)$ & $27 \%(15-44)$ & $5 \%(1-19)$ & 0.79 \\
FTT/Feeding problems & $32 \%(22-44)$ & $51 \%(36-67)$ & $5 \%(1-19)$ & 0.05 \\
\hline
\end{tabular}

$P$ values represent a comparison between BIT:s and Pre-BIT:s.
We saw increased referral to outpatient specialists as demonstrated by the larger number of well visits in the BIT: $\mathrm{s}$ group. We were unable to show a decrease in sick visits, because these were not reliably captured in the electronic medical records system. Infants with sBPD have a high incidence of postnatal growth failure prior to discharge which adversely affects lung development $[11,16]$. Up to $50 \%$ of children with BPD have a weight less than the 10th percentile at discharge $[16,17]$, a finding that was mirrored in our historical control group. After the creation of the multidisciplinary team, infants had better weight gain as demonstrated by their z-score at discharge. Further evidence of this impact can be found in the lower number of patients with failure to thrive (FTT) or feeding intolerance after discharge in the BIT:s era. Early referral to the outpatient gastrointestinal clinic and associated feeding team not only improved their objective weight gain, but subjectively improved the quality of the family's lives, as difficulties with feeding and weight gain is often cited by parents as one of their leading concerns [18]. In the first five years of life, we saw a reduction in the number of patients with an asthma diagnosis $(20 \%$ vs $37 \% p=0.06)$, but not in the albuterol prescriptions $(64 \%$ vs $65 \% p=0.9)$. It is unclear if this is a true difference, given that we did not see a difference in the number of ED visits, or readmissions for respiratory illnesses (data not reported). We feel confident that we have captured all of the readmissions and subspecialty visits as we are the only children's hospital in the state and the only hospital that provides subspecialty services to children.

The limitations of the study were the small sample size use of historical controls and retrospective nature. All patients in the Pre-BIT:s group had five years of follow up collected. Because not all of the patients in the BIT:s and moderate BPD groups had been alive for five years at the end of 2016 we limited our analysis of readmissions, ED and outpatient visits to within the first 2 years of life to try and statistically account for this difference between the groups. The limitation of the multidisciplinary BIT:s team is that it is primarily based in the NICU without an outpatient home. There is wide recognition that medically complex patients account for a disproportionate amount of hospital ED visits and admissions and that medical homes may help combat this trend [19].

\section{Conclusion}

The creation of a multidisciplinary team improved the inpatient outcomes of children with SBPD.

We shortened the NICU length of stay significantly without increasing re-admissions. While there have been many other advances made in the care of premature infants we think that this difference is in large part attributable to the creation of our multidisciplinary team. Weight gain, feeding difficulties and outpatient referrals to specialists were better in the BIT:s group, but these health effects did not appear to have long-lasting effects. We did not decrease the overall readmission rate in the first two years of life or change the percentage of common co-morbid conditions such as pulmonary hypertension. This is our first iteration of this team and we have already seen some success. More work needs to be done to create an outpatient care center that is able to consult on these patients when they are in the ED or re-admitted to see if we can improve long term outcomes and decrease health care utilization.

Acknowledgements We would like to acknowledge the William and Mary Oh - William and Elsa Zopfi Professorship in Pediatrics for Perinatal Research that helped provide funding for this study.

Funding William and Mary Oh - William and Elsa Zopfi Professorship in Pediatrics for Perinatal Research.

Author contributions RLM and MK conceptualized and designed the study, drafted the initial manuscript, and reviewed and revised the manuscript. JJS and ALB helped design the data collection instruments, collected data, and reviewed and revised the manuscript. JTM and $\mathrm{PH}$ prepared the statistics and reviewed and revised the manuscript. All authors approved the final manuscript as submitted and agree to be accountable for all aspects of the work. 


\section{Compliance with ethical standards}

Conflict of interest The authors declare that they have no conflict of interest.

Publisher's note Springer Nature remains neutral with regard to jurisdictional claims in published maps and institutional affiliations.

\section{References}

1. Doyle LW, Ford G, Davis N. Health and hospitalistions after discharge in extremely low birth weight infants. Semin Neonatol. 2003;8:137-45.

2. Bhandari A, Panitch HB. Pulmonary outcomes in bronchopulmonary dysplasia. Semin Perinatol. 2006;30:219-26.

3. Greenough A, Alexander J, Boit P, Boorman J, Burgess S, Burke A, et al. School age outcome of hospitalisation with respiratory syncytial virus infection of prematurely born infants. Thorax. 2009;64:490-5.

4. Stoll BJ, Hansen NI, Bell EF, Walsh MC, Carlo WA, Shankaran $\mathrm{S}$, et al. Trends in care practices, morbidity, and mortality of extremely preterm neonates, 1993-2012. JAMA. 2015;314:1039.

5. Farstad T, Bratlid D, Medbø S, Markestad T. Bronchopulmonary dysplasia - prevalence, severity and predictive factors in a national cohort of extremely premature infants. Acta Paediatrica. 2011;100:53-8.

6. Group E. Incidence of and risk factors for neonatal morbidity after active perinatal care: extremely preterm infants study in Sweden (EXPRESS). Acta Paediatrica. 2010;99:978-92.

7. Collaco J, Austin E, Zhang H, Baker C, Gien J, Cuevas Guaman M. Point prevalence, clinical characteristics, and treatment variation for infants with severe bronchopulmonary dysplasia. Am J Perinatol. 2015;32:960-7.

8. Padula MA, Grover TR, Brozanski B, Zaniletti I, Nelin LD, Asselin $\mathrm{JM}$, et al. Therapeutic interventions and short-term outcomes for infants with severe bronchopulmonary dysplasia born at $<32$ weeks' gestation. J Perinatol. 2013;33:877-81.

9. Ehrenkranz RA. Validation of the National Institutes of Health Consensus Definition of Bronchopulmonary Dysplasia. Pediatrics. 2005; 116:1353-60.

10. Jobe AH, Bancalari E. Bronchopulmonary dysplasia. Am J Respir Crit Care Med. 2001;163:1723-9.

11. Abman SH, Collaco JM, Shepherd EG, Keszler M, Cuevas-Guaman $\mathrm{M}$, Welty SE, et al. Interdisciplinary care of children with severe bronchopulmonary dysplasia. J Pediatrics. 2017;181:12-28.e1.

12. Mirza H, Ziegler J, Ford S, Padbury J, Tucker R, Laptook A. Pulmonary hypertension in preterm infants: prevalence and association with bronchopulmonary dysplasia. J Pediatrics. 2014;165: 909-14.e1.

13. Mousa H, Machado R, Orsi M, Chao CS, Alhajj T, Alhajj M, et al. Combined multichannel intraluminal impedance-pH (MII-pH): multicenter report of normal values from 117 children. Curr Gastroenterol Rep. 2014;16:400.

14. Landry JS, Menzies D. Occurrence and severity of bronchopulmonary dysplasia and respiratory distress syndrome after a preterm birth. Paediatrics Child Health. 2011;16:399-403.

15. Davis T, Meyer A, Beste J, Batish S. Decreasing low acuity pediatric emergency room visits with increased clinic access and improved parent education. J Am Board Fam Med. 2018;31:550-7.

16. Natarajan G, Johnson YR, Brozanski B, Farrow KN, Zaniletti I, Padula MA, et al. Postnatal weight gain in preterm infants with severe bronchopulmonary dysplasia. Am J Perinatol. 2014;31: 223-30.

17. Guimarães H, Rocha G, Guedes MB, Guerra P, Silva AI, Pissarra S. Nutrition of preterm infants with BPD after hospital discharge - Part 1. J Pediatric Neonatal Individ Med. 2014;3: e030116.

18. Pridham KF, Martin R, Sondel S, Tluczek A. Parental issues in feeding young children with bronchopulmonary dysplasia. J Pediatr Nurs. 1989;4:177-85.

19. Kuo DZ, Houtrow AJ. Recognition and management of medical complexity. Pediatrics. 2016;138:e20163021. 